BMJ Open Sport \& Exercise Medicine

\title{
Phosphorus supplementation raised the heart rate of male water polo players during a randomised graded dryland exercise test
}

\author{
Rami Elhusseini (1) , Elie-Jacques Fares, Omar Obeid
}

\begin{abstract}
To cite: Elhusseini R, Fares E-J, Obeid 0. Phosphorus supplementation raised the heart rate of male water polo players during a randomised graded dryland exercise test. BMJ Open Sport \& Exercise Medicine 2020;6:e000714. doi:10.1136/ bmjsem-2019-000714
\end{abstract}

- Additional material is published online only. To view, please visit the journal online (http://dx.doi.org/10.1136/ bmjsem-2019-000714)

Accepted 22 March 2020

\section{ABSTRACT}

Objective The impact of phosphorus supplementation on athletic performance is unclear. Ingestion of phosphorus for several days has been reported to increase cardiac capacity, improve oxygen muscle kinetics and enhance lactate buffering capacity. Recent studies have shown that phosphorus ingestion with a meal increases postprandial glucose uptake and thermogenesis. The present study aimed to assess the effect of acute phosphorus ingestion with a meal on specific workload parameters.

Methods A double-blind, crossover trial of 12 male water polo players between 18 and 22 years old was conducted. Overnight fasted subjects were asked to cycle for $20 \mathrm{~min}$ before ingesting $100 \mathrm{~g}$ of glucose with phosphorus or placebo $(400 \mathrm{mg})$. Three hours later, they were asked to perform a graded cycling exercise for $25 \mathrm{~min}$.

Results Expenditure, respiratory quotient, perception of fatigue and exercise efficiency were similar between treatments. However, heart rate was significantly higher in the phosphorus group ( $142 \pm 10$ beats/min) compared with placebo (135 \pm 10 beats/min).

Conclusion Exercise performance 3 hours after the coingestion of glucose with phosphorus did not affect substrate use, while heart rate was increased. The heart rate increase could be attributed to a rise in core body temperature.

Trial registration number NCT03101215.

\section{BACKGROUND/RATIONALE}

The ergogenic benefits of phosphorus supplementation have been explored since the beginning of the 20th century, but the results are often ambivalent. ${ }^{12}$ In most studies, the effect of chronic phosphorus loading, 2-3 g/ day, was studied. ${ }^{3}$ The impact of phosphorus was thought to be its capacity to increase plasma 2-3diphosphoglycrate (2,3 DPG), which reduces $\mathrm{O}_{2}$ affinity to haemoglobin. We recently found that phosphorus coingestion with carbohydrates led to an increase in postprandial intracellular glucose uptake. ${ }^{4}$ Phosphorus is also known to enhance glycogenolysis and glycogenesis, since it is needed for glycogen phosphorylase activity, and ATP

\section{What are the new findings?}

- Phosphorus supplementation increased heart rate during exercise performed 3 hours later.

- Acute phosphorus supplementation has a significant metabolic effect.

- Phosphorus supplementation does not affect the breathing rate, perception of fatigue or exercise efficiency. 1

How might it impact on clinical practice in the near future?

Use of phosphorus supplementation in competitions taking place in cold water as possible protection from hypothermia.

production. $^{5-7}$ In most studies, phosphorus was not ingested with the meal, which may have reduced its impact. ${ }^{5-8}$ In one study, acute supplementation using calcium phosphate, but administered without a meal, failed to detect an effect on athletic performance. ${ }^{8}$ However, the impact of acute phosphorus ingestion with a meal on athletic performance remains to be elucidated especially since phosphorus coingestion with a meal is known to affect glycogen and ATP statuses. The acute dose also helps in isolating its metabolic effect on glycogen alone rather than 2,3 DPG known to be affected by chronic intake, making it a confounding variable. ${ }^{9-11}$

\section{Objective}

The objective of this study was to investigate the ergogenic effect of acute phosphorus (400 mg) ingestion with $100 \mathrm{~g}$ glucose using male water polo players.

\section{Methods}

Experimental protocol

A crossover double-blinded study was conducted on male water polo players. Neither the participants nor the person in 
charge of conducting the test knew which treatment sequence was being administered. Only the lab assistant had access to the randomised AB-BA setup recorded separately. Choosing members of the water polo team with similar training schedules allowed for a relatively homogenous response to prolonged exercise and perception of fatigue. ${ }^{12-14}$ The training routine starts with warm-up laps of $50 \mathrm{~m}$ for $5 \mathrm{~min}$, followed by $15 \mathrm{~m}$ sprints/underwater dive sequences for $10 \mathrm{~min}$ that include $10 \mathrm{~s}$ rest intervals between bouts, and reaching an estimated $95 \%$ of maximum heart rate capacity. Another 5 min are spent doing water treading, which consists of holding both arms at shoulder level while doing eggbeaters. Eggbeater is a specific water polo leg movement technique consisting of alternate breaststroke kicks. ${ }^{15}$ This strenuous routine done two times a week makes the sample relatively similar in exercise tolerance and perception of fatigue.

The study consisted of two visits to the metabolic lab at the Department of Nutrition and Food Sciences, where all data were collected; in each visit, the subject received the placebo or phosphorus treatment in a random manner. The protocol of each visit was as follows:

1. Overnight fasted participants were received at the testing facility (Department of Nutrition and Food Sciences) in the morning; they underwent a body composition analysis using bioelectrical impedance analysis (InBody brand 770 model) and were asked to rest for $30 \mathrm{~min}$ before wearing the heart rate monitor and gas exchange mask using the indirect calorimeter (Quark, CPET; Cosmed, Rome, Italy) in order to determine baseline energy expenditure and heart rates.

2. After baseline measurements, participants were asked to cycle on the ergometer for $20 \mathrm{~min}$ at an average of $70 \%$ of the maximal heart rate while wearing the mouthpiece, to be familiar with the process, and the Borg scale ${ }^{16}$ was introduced.

3. After baseline measurements, participants were asked to cycle on the ergometer for $20 \mathrm{~min}$ at an average of $70 \%$ of the maximal heart rate while wearing the mouthpiece, to be familiar with the process, and the Borg scale ${ }^{16}$ was introduced.

4. After baseline measurements, participants were asked to cycle on the ergometer for $20 \mathrm{~min}$ at an average of $70 \%$ of the maximal heart rate while wearing the mouthpiece, to be familiar with the process, and the Borg scale ${ }^{16}$ was introduced.
5. Cycling started at $75 \mathrm{~W}$ and an average of 85 revolutions per minute (RPM) was maintained throughout the test. The load was increased by $25 \mathrm{~W}$ every $5 \mathrm{~min}$. In case the participants were unable to reach $150 \mathrm{~W}$, an increment of $10 \mathrm{~W}$ was used instead. The perception of fatigue was recorded at the last minute of each 5 min stage using the $6-20$ Borg scale. ${ }^{16}$

During the workload test, several parameters were tested, including heart rate, energy expenditure, exercise efficiency, respiratory quotient, breathing rate and perception of fatigue.

\section{Statistical design}

Sample size

Sample size was estimated based on previous studies, varying from 6 to 11 participants. Since supplementation is relatively safe, especially at the low doses we administer, and because any improvement is valuable, we opted for a Power of $60 \%\left(\mathrm{Z}_{\beta}=0.84\right)$, and a $10 \%$ probability of false positive $\left(Z_{\alpha}=1.64\right)$. Sample size calculation for two unpaired samples with an estimated 10 beats $/ \mathrm{min}$ difference, requires a sample of 10 (using the following link: https://select-statistics.co.uk/calculators/sample-sizecalculator-two-means/.

\section{Statistical methods}

The unpaired t-test for two means was used based on the recommendation of Wellek and Blettner, ${ }^{17}$ since it is a crossover sample with randomised AB-BA assignment of treatment (online supplementary files 1 and 2). Carryover effect was estimated using the aforementioned method ( $\mathrm{T}$ score $=-0.397, \mathrm{p}$ value $=0.7006)$. A column indicating carry over T-score and $\mathrm{p}$ value were added to the tables.

\section{RESULTS}

The workload test showed that energy expenditure increase by about $10 \mathrm{kcal} / \mathrm{min}$ over the test period (from a rest value of about $2 \mathrm{kcal} / \mathrm{min}$ until more than $12 \mathrm{kcal} /$ $\min$ ), and this increase was similar between the two treatments and thus not affected by phosphorus addition to the glucose load (table 1). Moreover, exercise efficiency during the workload test of the phosphorus treatment $(21.0 \pm 2.2)$ was similar to that of the placebo $(21.2 \pm 2.5)$.

The average heart rate in beats per minute at rest or before the start of the workload test was similar between the two treatments, while the heart rate of the phosphorus

Table 1 Energy expenditure ( $\mathrm{kcal} / \mathrm{min})$ of subjects after the four stages of workload

\begin{tabular}{lllllll}
\hline Workload & $\mathbf{n}$ & Phosphorus & Placebo & P value & Carry over T score & Carry over P value \\
\hline Rest & 11 & $2.063 \pm 0.316$ & $1.961 \pm 0.219$ & 0.146 & 1.093 & 0.302 \\
At 75 W & 12 & $8.174 \pm 1.165$ & $7.916 \pm 1.085$ & 0.128 & 0.702 & 0.498 \\
At 100 W & 12 & $9.586 \pm 1.032$ & $9.542 \pm 0.993$ & 0.788 & 0.659 & 0.524 \\
At 125 W & 12 & $11.18 \pm 1.018$ & $11.13 \pm 1.126$ & 0.798 & 0.240 & 0.815 \\
At 150 W & 12 & $12.95 \pm 1.138$ & $12.73 \pm 1.205$ & 0.078 & -0.0585 & 0.954 \\
\hline
\end{tabular}


Table 2 Heart rate (beats/min) of subjects after the four stages of workload

\begin{tabular}{lccclll}
\hline Workload & $\mathbf{n}$ & Phosphorus & Placebo & P value & Carry over T score & Carry over P value \\
\hline At rest (fasted) & 11 & $74 \pm 5$ & $73 \pm 5$ & 0.688 & 0.367 & 0.722 \\
At rest & 10 & $82 \pm 8$ & $78 \pm 6$ & 0.105 & 1.493 & 0.170 \\
At 75 W & 11 & $119 \pm 9$ & $111 \pm 8$ & 0.0036 & 0.786 & 0.452 \\
At 100 W & 11 & $134 \pm 9$ & $127 \pm 8$ & 0.000086 & -0.428 & 0.678 \\
At 125 W & 11 & $149 \pm 10$ & $142 \pm 10$ & 0.00047 & -0.793 & 0.448 \\
At 150 W & 11 & $166 \pm 12$ & $160 \pm 13$ & 0.00047 & -0.470 & 0.649 \\
Average heart rate & 11 & $142 \pm 9$ & $135 \pm 9$ & 0.000017 & -0.397 & 0.7006 \\
\hline
\end{tabular}

treated group maintained a significantly higher value throughout the testing period. During the work load test, the heart rate of the phosphorus treatment averaged at $142 \pm 9$ compared with $135 \pm 9$ for the placebo treatment $(\mathrm{p}$ value $<0.001$ ) (table 2 ).

\section{DISCUSSION}

The ability of phosphorus to stimulate postprandial glycogen synthesis ${ }^{7}$ as well as glycogenolysis (through stimulation of glycogen phosphorylase) incited us to investigate the impact of phosphorus on workload, known to be affected by glycogen status. ${ }^{18}$ The dose of $400 \mathrm{mg}$ was chosen to remain within one-third of phosphorus recommended dietary allowance (RDA) for males aged 18-50 years old, ${ }^{19}$ and minimise the carryover effect, also minimised by adopting a weeklong washout period. This was verified statistically using the methodology described by Wellek and Blettner. ${ }^{17}$ Period effect was minimised by scheduling treatment sequence (AB-BA) at the same time of day, and no more than 2 weeks apart, to account for the circadian phosphorus level. ${ }^{20}$ The bolus dosing regime was adopted to detect the effect of supplementing $1 \mathrm{mg}$ phosphorus per kcal, noted to be the sufficient rate to detect an effect in previous phosphorus supplementation studies. ${ }^{41}$ The population is composed of team members with similar training routine, which leads to a relatively similar response to prolonged exercise and intensity of workload. Water polo is known for its harsh training conditioning, as described in the methodology, making our population likely to have a lower score on the perception of fatigue scale. The other distinctive feature is the ability to perform intensive exercise in water, compared with leisurely swimming, and the likelihood to practice in a lake or non-heated pool ahead of ideal water temperature season.

Phosphorus addition to food did not affect energy expenditure of the workload test, although it had been reported to raise postprandial energy expenditure (0-4hours after meal $)^{22}$ probably to enhance the postprandial anabolic processes. The heart rate increase during exercise points towards a rise in core temperature. ${ }^{23}$

The similarity in heart rate between the treatment groups before the workload test implies that phosphorus ingestion did not affect the cardiac rhythm. The ability of an acute phosphorus dose to increase heart rate during a workload test may be related to a potential increase in core temperature. The increase in core temperature may be related to the capacity of phosphorus to raise postprandial thermogenesis. $^{22}$ Furthermore, the observed increase in heart rate may have been an adaptive mechanism to compensate for a decrease in exercise efficiency. Phosphorus, on the other hand, seems to induce an increase in heat shock protein synthesis, which protects eukaryotic cells against temperature elevation, since phosphorus-deficient animals were found unable to produce the required homeostatic compensation during heat stress. ${ }^{24} 25$ This study showed that increased heart rate did not have an effect on exercise efficiency (table 3). A curious finding since an increased heart rate normally leads to a decrease in exercise efficiency and to increased fatigue. ${ }^{10}$ A possible direction of our research in sports nutrition can focus on the effect of acute phosphorus supplementation on efficiency in water polo players swimming in non-heated pools or in open water, in line with researching the effect of new practices in competitive sports. ${ }^{2627}$ Since many teams start their open water training when the water temperature is relatively lower than traditional pools where temperature is set to $26^{\circ} \mathrm{C},{ }^{28}$ the increase in core temperature could provide an ergogenic advantage.

\section{CONCLUSION}

In water polo players, the addition of phosphorus to a glucose load failed to affect subsequent energy expenditure during a workload test performed 3 hours later. At the same time, exercise efficiency was not affected despite a significant increase in heart rate.

Out of 14 participants, 12 had their energy expenditure per minute (EEm) measured following the four-increment protocol; 1 participant followed two different protocols;and

\begin{tabular}{llllllll}
\hline Table 3 & \multicolumn{1}{l}{ Exercise efficiency $(\%)$ of subjects after the four stages of workload } & \\
\hline Exercise efficiency & $\mathbf{n}$ & Phosphorus & Placebo & P value & Carry over T score & Carry over P value \\
\hline & 13 & $21.0 \pm 2.2$ & $21.2 \pm 2.5$ & 0.767 & 1.672 & 0.123 & \\
\hline
\end{tabular}


1 participant had a mask malfunction, requiring us to omit his EEm value. One participant did not have a resting EEm measurement; therefore, the energy expenditure at rest was calculated in 11 participants. There was no significant increase in energy expenditure at any workload and no significant increase throughout all stages of the trial.

Overnight fasted subjects were asked to cycle for $20 \mathrm{~min}$ before being given $100 \mathrm{~g}$ of glucose with or without phosphorus $(400 \mathrm{mg})$. Three hours later, they were ask to perform a graded cycling exercise for $25 \mathrm{~min}$, and both measures energy expenditure and heart rate were monitored.

Eleven participants' data for heart rate were analysed. Two subjects' HR measurements during the rest period on the bike before the start of pedalling were not registered.

Participants $(n=13)$ had their exercise efficiency calculated from four different points of the EEm curve; each phase of the four time trial stages consisted of pedalling for $5 \mathrm{~min}$ at a constant speed and a constant load. We measured the EEm after the third minute to be sure it reflects the adapted metabolic effort. No significant difference in energy efficiency was detected in 13 crossover pairs after phosphate supplementation.

Correction notice The article has been corrected since it was published online. The summary box has been updated.

Twitter Rami Elhusseini @ramilhusseini

Contributors E-JF: data analysis, design of protocol and literature review. RE: trial design, data collection and analysis, literature review and writing of the manuscript. 00: advisor, literature review, editing and design.

Funding The authors have not declared a specific grant for this research from any funding agency in the public, commercial or not-for-profit sectors.

Competing interests None declared.

Patient consent for publication Not required.

Ethics approval Institutional review board approval (NUT.00.24) was granted by the Human Research Protection Program and Institutional Review Board, Clinical Research Institute, American University of Beirut. The experimental protocol was approved by the American University of Beirut's Institutional Review Board.

Provenance and peer review Not commissioned; externally peer reviewed.

Data availability statement All data relevant to the study are included in the article or uploaded as supplementary information.

Open access This is an open access article distributed in accordance with the Creative Commons Attribution Non Commercial (CC BY-NC 4.0) license, which permits others to distribute, remix, adapt, build upon this work non-commercially, and license their derivative works on different terms, provided the original work is properly cited, appropriate credit is given, any changes made indicated, and the use is non-commercial. See: http://creativecommons.org/licenses/by-nc/4.0/.

ORCID iD

Rami Elhusseini http://orcid.org/0000-0003-4337-8399

\section{REFERENCES}

1 Buck CL, Wallman KE, Dawson B, et al. Sodium phosphate as an ergogenic aid. Sports Med 2013;43:425-35.

2 West JS, Ayton T, Wallman KE, et al. The effect of 6 days of sodium phosphate supplementation on appetite, energy intake, and aerobic capacity in trained men and women. Int J Sport Nutr Exerc Metab 2012;22:422-9.
3 Kopec BJ, Dawson BT, Buck C, et al. Effects of sodium phosphate and caffeine ingestion on repeated-sprint ability in male athletes. $J$ Sci Med Sport 2016;19:272-6.

4 Khattab M, Abi-Rashed C, Ghattas H, et al. Phosphorus ingestion improves oral glucose tolerance of healthy male subjects: a crossover experiment. Nutr J 2015;14:112.

5 Chasiotis D. Role of cyclic AMP and inorganic phosphate in the regulation of muscle glycogenolysis during exercise. Med Sci Sports Exerc 1988;20:545-50.

6 Hargreaves M, Richter EA. Regulation of skeletal muscle glycogenolysis during exercise. Can J Sport Sci 1988;13:197-203.

7 Mattar LE, Mattar MA, Batal M, et al. Stimulation of postprandial in vivo glycogenesis and lipogenesis of rats fed high fructose diet with varied phosphate content. Nutr Res 2010;30:151-5.

8 Galloway SD, Tremblay MS, Sexsmith JR, et al. The effects of acute phosphate supplementation in subjects of different aerobic fitness levels. Eur J Appl Physiol Occup Physiol 1996;72:224-30.

9 Ivy JL. Muscle glycogen synthesis before and after exercise. Sports Med 1991;11:6-19.

10 Ørtenblad N, Westerblad H, Nielsen J. Muscle glycogen stores and fatigue. J Physiol 2013;591:4405-13.

11 Rauch HGL, St Clair Gibson A, Lambert EV, et al. A signalling role for muscle glycogen in the regulation of PACE during prolonged exercise. Br J Sports Med 2005;39:34-8.

12 Price TB, Sanders K. Muscle and liver glycogen utilization during prolonged lift and carry exercise: male and female responses. Physiol Rep 2017;5:e13113.

13 Botonis PG, Toubekis AG, Terzis GD, et al. Performance decrement and skill deterioration during a water polo game are linked with the conditioning level of the athletes. J Strength Cond Res 2016;30:1033-41.

14 Lobenius K. Physiological differences during exercise in water and on cycle ergometer for synchronised swimmers. Saint-Etienne: University of Saint-Etienne, 2003: 539-44.

15 Sanders RH. Analysis of the Eggbeater kick used to maintain height in water polo. J Appl Biomech 1999;15:284-91.

16 Borg E, Kaijser L. A comparison between three rating scales for perceived exertion and two different work tests. Scand J Med Sci Sports 2006;16:57-69.

17 Wellek S, Blettner M. On the proper use of the crossover design in clinical trials: Part 18 of a series on evaluation of scientific publications. Dtsch Arztebl Int 2012:109:276-81.

18 Arnall DA, Nelson AG, Quigley J, et al. Supercompensated glycogen loads persist 5 days in resting trained cyclists. Eur J Appl Physiol 2007;99:251-6

19 Institute of Medicine (US) Standing Committee on the Scientific Evaluation of Dietary Reference Intakes. Dietary reference intakes for calcium, phosphorus, magnesium, vitamin $D$, and fluoride. Washington, DC: National Academies Press (US), 1997.

20 Song C, Wang J, Kim B, et al. Insights into the role of circadian rhythms in bone metabolism: a promising intervention target? Biomed Res Int 2018;2018:1-11.

21 Obeid OA, Dimachkie S, Hlais S. Increased phosphorus content of preload suppresses ad libitum energy intake at subsequent meal. Int $J$ Obes 2010;34:1446-8.

22 Assaad M, El Mallah C, Obeid O. Phosphorus ingestion with a highcarbohydrate meal increased the postprandial energy expenditure of obese and lean individuals. Nutrition 2019;57:59-62.

23 Buller MJ, Tharion WJ, Cheuvront SN, et al. Estimation of human core temperature from sequential heart rate observations. Physiol Meas 2013:34:781-98.

24 Mahmoud KZ, Edens FW, Eisen EJ, et al. The effect of dietary phosphorus on heat shock protein mRNAs during acute heat stress in male broiler chickens (Gallus gallus). Comp Biochem Physiol C Toxicol Pharmacol 2004;137:11-18.

25 Staib JL, Tümer N, Powers SK. Increased temperature and protein oxidation lead to HSP72 mRNA and protein accumulation in the in vivo exercised rat heart. Exp Physiol 2009;94:71-80.

26 Melchiorri G, Castagna C, Sorge R, et al. Game activity and blood lactate in men's elite water-polo players. J Strength Cond Res 2010;24:2647-51.

27 Galy O, Ben Zoubir S, Hambli M, et al. Relationships between heart rate and physiological parameters of performance in top-level water polo players. Biol Sport 2014;31:33-8.

29 Federation Internationale de Natation Amateur. FINA handbook. Cincinnati, Ohio: Federation Internationale de Natation Amateur, 2018. 